\title{
Research on Optimization Model of Logistics Transportation Truck Path considering Environmental Impact: Experimental Data from Xiqing District, Tianjin
}

\author{
Qianqian Han, ${ }^{1}$ Yao Sun, ${ }^{2}$ Qingdong Wu $\mathbb{D}^{3},{ }^{3}$ and Zijian $\mathrm{Bai}^{2}$ \\ ${ }^{1}$ Business School, Qingdao University, Qingdao 266071, Shandong, China \\ ${ }^{2}$ Tianjin Municipal Engineering Design and Research Institute, Tianjin 300051, China \\ ${ }^{3}$ Shandong Luqiao Group Co., Ltd., Jinan 250021, Shandong, China \\ Correspondence should be addressed to Qingdong Wu; wuqingdong_qd@163.com
}

Received 27 October 2020; Revised 25 December 2020; Accepted 8 January 2021; Published 20 January 2021

Academic Editor: Ronghui Zhang

Copyright $(92021$ Qianqian Han et al. This is an open access article distributed under the Creative Commons Attribution License, which permits unrestricted use, distribution, and reproduction in any medium, provided the original work is properly cited.

In recent years, the prohibition of trucks which could cause environmental pollution on urban roads has become widespread in China. However, some truck restriction policies might lead to a reduction in logistics transportation efficiency. With the help of big data, technology companies have developed many truck applications, such as HCB, truck home, and truck help, to provide the drivers available traffic information. In this context, this paper put forward a truck path optimization model considering environmental impact (TPOM-EI), which is solved by a heuristic algorithm-ant colony optimization (ACO) algorithm. Most previous studies focused on unilateral benefits rather than overall benefits; this paper aims to propose a path optimization model based on real-time minimization of social and transportation costs. Finally, data of Xiqing Economic and Technological Development Zone in Tianjin city (XQ-EDZ) have been used to demonstrate the applicability of the proposed algorithm. The results show that logistics truck path has a huge impact on social costs, and real-time activities in various areas will also change the path of a truck. This research will also help logistics truck drivers to choose the best route in real time.

\section{Introduction}

With the rapid development of economy and the explosive growth of urban population in China, urban problems such as traffic congestion and environmental pollution have become increasingly serious, affecting the people's lives and the sustainable development of cities. Logistics transportation is an important factor causing the above problems. In order to solve these problems, traffic management department tried to limit the run paths of trucks, especially high-emission trucks, to reduce traffic congestion and environmental pollution. Many logistics transportation policies have been formulated, such as imposing fines on carbon-intensive roads, truck restrictions, and promoting clean energy trucks.

However, there are some deficiencies about the impact of logistics policies on logistics benefits in the current study. First of all, there is no definitive conclusion on whether urban logistics policies are conducive to solve problems such as urban congestion and urban environmental pollution in academia. Tamagawa et al. [1] have concluded through simulation experiments that the combined measures of truck restrictions and discounted tolls on highways have a huge effect on improving urban pollution emissions. Arvidsson [2] has proposed that increasing the full load rate instead increased truck exhaust emissions in severe pollution areas. Russo and Comi [3] put forward that the use of environmentally friendly vehicles is the best choice for achieving sustainable urban development in small- and medium-sized cities. Teo et al. [4] have studied the environmental benefits of charging an exhaust emission penalty policy for highemission trucks, and the results showed that this policy actually increased exhaust emissions.

Besides, we have found that the intensity of land development has been increasing, construction land has 
continued to expand beyond the original boundary, and urbanization has accelerated further. In the process, the traffic characteristics of suburban roads changed significantly. Due to the changes, suburban roads originally hold a lot of transit traffic and gradually transform to the shortdistance traffic. Suburban roads gradually have an urban road function, especially in development zone. The phenomenon of mixed passenger and freight traffic became increasingly serious, which caused frequent traffic accidents. These traffic accidents also brought about other problems such as noise and air pollution that made people complain.

At present, traffic management department only implements a traffic control measure for one or more closed areas from a static perspective. There is no detailed demonstration of how to plan the restricted areas scientifically. Related academic research achievements are few. If the restricted areas are too large, the transportation cost will be increased. On the contrary, it will not be able to effectively reduce the social cost (e.g., traffic safety, environmental pollution, etc.). The social cost and transportation cost of trucks traveling on different paths are also different. How to find the optimal truck path has become a key issue in the division of the restricted areas.

In the past research, most researchers have tended to minimize the traffic congestion and environmental pollution caused by logistics trucks with unilateral benefits rather than overall, which led to a reduction in logistics transportation efficiency [5-9]. Some researches lack quantitative evaluation and analysis using empirical methods in policy development. Usually, the methods are modeling and simulation, and the data sources come from enterprise surveys. That is why the policy formulation lacks flexibility, timeliness, and robustness.

Based on the above, we try to find a method to optimize logistics transportation truck path with the objective function of minimizing social and transportation costs in real time. The social costs contain the traffic congestion cost, emissions cost, and safety cost. Our research would provide solutions for dynamic dispatch of trucks by traffic management department. Besides, trucks are gradually connected to the Internet with the help of big data and Internet of Things technology, and some software such as "truck home" for truck navigation has appeared. It would provide practical application scenarios for our research.

\section{Literature Review}

As an important part of the urban transportation system, logistics transportation has a significant contribution to improving the quality of urban economic and social operations, but it will also bring about negative effects such as environmental pollution and traffic congestion [10]. In order to reduce the negative externalities generated by logistics transportation, the traffic management department usually implements traffic control measures for trucks entering the urban area. The most typical one is truck restrictions. Truck restrictions can significantly reduce the freight traffic flow in restricted areas (generally the central area), thereby effectively reducing the interference of trucks on the urban center shopping environment and traffic, which have better social benefits [11]. Meanwhile truck restrictions will cause trucks to enter, pass, and stop difficultly. That will increase the cost and efficiency of logistics transportation, affect the quality of distribution services [12], and even make logistics transportation become a shortcoming of urban logistics under the truck restriction policy [13]. In addition, truck restrictions may also cause new problems. For example, logistics transportation companies use passenger vehicles transport goods to reduce transportation costs, which will increase traffic volume and environmental pollution in restricted areas [14]. Related policies and strategies, models, and algorithms are reviewed in detail below.

2.1. Logistics Transportation Policies and Strategies. As early as 1948, the Journal of Economics published related research on "carrot and stick" policy, which divided the feedback of policy on object behavior into punishment and incentive. As far as urban logistics transportation policies are concerned, many cities generally adopt punitive policies, such as penalties for fines for trucks that enter the specified area of a nonspecified time or for trucks that exceed the standard. The purpose of implementing the urban logistics penalty policy is to alleviate urban traffic congestion and environmental pollution. Urban traffic congestion and environmental pollution are considered to be largely caused by logistics transportation. According to the statistics, delays and other losses caused by traffic congestion amount to $1 \%$ of EU's overall GDP [15].

In the 1980s, in the Italian capital of Rome, an area of five square kilometers is designated as the central restricted traffic zone. This area restricts the entry and exit of trucks unless paying an entry fee [16]. Santiago implemented an annual traffic restriction policy on vehicle control in 2008, that is, from April to August in the winter (Southern Hemisphere), restricting $40 \%$ of vehicles without exhaust catalysts from entering the urban area to reduce emissions [17].

Since their development, many cities around the world have implemented freight restrictions. In Germany, government and urban planning departments have formulated a number of truck restrictions for decades, such as levying highway tolls for trucks which load more than 12 tons in order to reduce the externalities brought by logistics transportation [18]. In Ahmedabad of India, the urban transport policy department has established controls on trucks to restrict them into and out of the restricted area except from $11 \mathrm{pm}$ to 7 am in the next day or from $1 \mathrm{pm}$ to 4 pm [19]. There are also logistics truck restrictions due to temporary international activities. During the 2012 Olympic Games in London, the United Kingdom imposed temporary restrictions on trucks. It generally claims that logistics transportation activities should run outside normal operating hours [20].

In Beijing, truck restrictions were due to road maintenance and temporary foreign affairs in the early years. Recently, department of traffic management has launched a series of policies and strategies to reduce 
congestion pressure with the increasing number of vehicles. Since 2008, it has been forbidden to enter the roads within the Fourth Ring Road from 6 am to $11 \mathrm{pm}$, and trucks over 8 tons are forbidden to enter Fifth Ring Road from 6 am to $10 \mathrm{pm}$ in Beijing. Other cities in China have begun to implement plate number restrictions since 2010, such as Guangzhou, Shanghai, Tianjin, and Qingdao $[21,22]$.

2.2. Exhaust Emission Model. One of the most important reasons to restrict logistics transport truck is severe pollution caused by its exhaust emissions. Therefore, the study of exhaust emission model can evaluate the effectiveness of the truck restrictions. Many scholars have researched on the quantitative evaluation of vehicle exhaust emissions and developed a variety of models for quantitative evaluation of exhaust emissions. According to the model's application level and development ideas, the existing exhaust emission models can be divided into three levels: macro, meso, and micro.

Macroscopic exhaust emissions evaluation uses the emission factor based on average speed as a calculation parameter to calculate and evaluate the tail gas emissions. Macro emission models include the MOBILE model developed by the US Environmental Protection Agency [23], the EMFAC model developed by the California Air Resources Agency [24], and the COPERT model developed by the European Environment Agency on their own [25]. Mesoscale models are mainly used to analyze and evaluate exhaust emissions in lanes, traffic communities, and other areas. Typical mesoscale exhaust models include MEASURE model and VT-Micro model. The microexhaust model could analyze the emissions of specific areas, roads, or intersections and evaluate real-time exhaust emissions of motor vehicles. Typical microexhaust models include CMEM model and ONRORD model.

Sugawara and Niemeier [26] obtained the exhaust emissions at different average vehicle speed using MOBILE model as the optimal objective function value. Ericsson et al. [27] studied the path selection behavior of travelers by extracting basic traffic data from Delong, Sweden, with the goal of minimizing transportation fuel and exhaust emissions. Kyoungho and Hesham [28] analyzed the actual selection behavior of motor vehicle drivers and found that some drivers choose the shortest path in order to save time or avoid congestion. Jaeyoung et al. [29] combined the microsimulation model TRANSIMS with the microenergy consumption model VT-Micro and applied genetic algorithms to study the time consumption, energy consumption, and emissions of transportation corridors.

2.3. Path Optimization Algorithm. In 1959, Dutch computer scientist Dijkstra proposed the Dijkstra algorithm [30]. Its main idea is to construct a path tree by increasing the length of the path and develop the leaf nodes of the path tree to obtain the shortest path from the root node to the leaf node path. In 1962, Floyd [31] proposed the Floyd algorithm. The algorithm constructed the shortest path matrix of each two points in the graph through the weight matrix of the graph. These algorithms are classic path optimization algorithm.

As early as 1959, Dantzig and Ramser proposed the vehicle routing problem (VRP), which belongs to NP-hard problem [32]. Solving VRP problems can be divided into precise algorithms and heuristic algorithms. Only when the problem size is small can accurate algorithms find the optimal solution in an acceptable time. There is not much research on the precise solution of VRP. The most representative is the branch pricing cutting algorithm proposed by Dinh et al. [33]. Subsequently, Ren et al. further improved the algorithm performance by using a strategy of increasing the number of cuts and proposed the precise set partitioning algorithm [34]. In this paper, we would solve a truck route problem considering environmental impact. Since this problem is more complicated than the classical vehicle path, heuristic algorithms are often used for solving it. The more commonly used algorithms include particle swarm optimization, ant colony algorithm, and parallel tabu search heuristic method [35-37].

In this paper, we aim to find the optimal truck paths considering transportation costs and social costs for solving the real-time logistics transportation scheduling. The remainder of the paper is organized as follows. Section 3 shows the description of the problem and develops a model to design truck paths based on ant colony algorithm. In Section 4 , we present the process of the algorithm. A case study in Tianjin city of China is present in Section 5. At last, the conclusions are provided in Section 6.

\section{Model Foundation}

3.1. Problem Description. At present, many cities have implemented truck restrictions strategy in order to reduce regional environmental pollution, traffic congestion, and safety issues. But it is more appropriate to say that the strategy would reduce the people's loss in the restricted zone. Trucks passing through different areas can cause unequal loss on people in the restricted zone. The unequal loss is social costs which contain congestion cost, emissions cost, and safety cost. The loss is also proportional to the amount of activity in different areas. According to statistics surveys of urban residents, the tolerance of trucks in commercial and residential areas is significantly lower than that in industrial areas.

As shown in Figure 1, the truck departs from origin point $O$ and reaches destination point $D$ in Sioux Falls network. There are 24 nodes in the network and the lines represent routes. All the routes divide the restricted zone into 14 areas. Each area has different land use properties. When a truck is passing through a route, it will have a negative impact on the passed areas which are enclosed by the route. The truck that passes through route $i, j$ is denoted by $(i, j)$. Assume that the path of the truck's $O D$ is the bold green line. When the truck passes route $(O, 2)$, it only affects the people in area 1 , while it would affect the people in areas 1 and 2 when the truck passes route $(2,3)$. Each area could calculate the whole loss $S$ and each route has a certain loss. Take route $(13,14)$ as an 


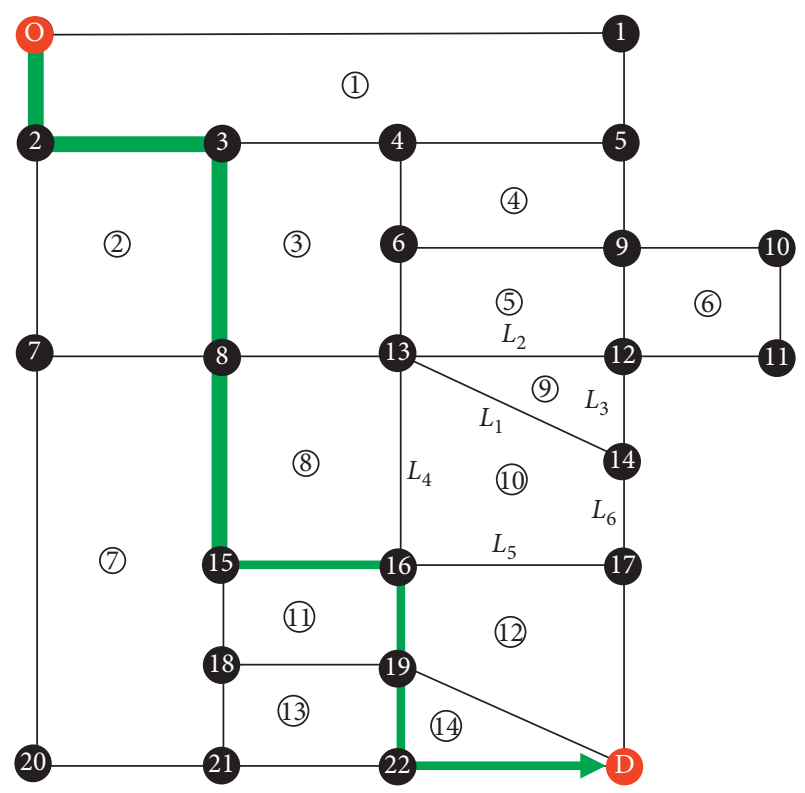

Figure 1: Logistics transportation truck path optimization diagram.

example; the total loss of people in areas 9 and 10 can be expressed as

$$
S_{13,14}=\frac{1}{2}\left(\frac{L_{1} \cdot S_{9}}{L_{1}+L_{2}+L_{3}}+\frac{L_{1} \cdot S_{10}}{L_{1}+L_{4}+L_{5}+L_{6}}\right),
$$

where $S_{13,14}$ denotes the loss when the truck passes route (13, 14), $S_{9}$ and $S_{10}$ denote the loss of areas 9 and area 10 , respectively, and $L$ denotes the length of each route.

The activity and congestion are different for each area at a different time horizon. If we do not consider the social cost, the truck should choose the shortest path to pursue maximum benefits. However, it could have a serious impact on traffic congestion, environmental pollution, and safety in dense areas. The truck should avoid high-impact areas to reduce social costs. In summary, we establish a model considering the environmental impact and area property to minimize social costs and transportation costs. The specific optimization model is given in Section 3.2.

\subsection{Truck Path Optimization Model considering Environ-} mental Impact (TPOM-EI). Logistics transportation truck running on urban roads has many negative impacts on local residents. Firstly, compared with private cars, logistics transportation truck runs slower with longer bodies that there would be higher probability of causing traffic congestion. The book of Highway Capacity Manual shows that the standard car conversion coefficient of a truck is 4 times that of a private car. Secondly, logistics transport truck produces more emissions, especially when it is running on urban roads with frequent deceleration and idling. Finally, it has poor drivability due to its heavy weight and causes traffic accidents in high-density areas. Therefore, we select traffic congestion, exhaust emission, and traffic safety as the assessment of the increased social costs brought by logistics transportation truck. Truck path optimization model considering environmental impact (TPOM-EI) has been put forward in this paper.

3.2.1. Traffic Congestion Cost. Logistics transportation truck could exacerbate traffic congestion [38]. Due to the characteristics of a large body and low speed, it is difficult for private cars to overtake during peak hours, which become a bottleneck on urban road. Let TC be the traffic congestion cost; it is a measure of the marginal congestion cost of trucks traveling on urban road.

$$
\begin{aligned}
\mathrm{TC} & =\sum_{i} \sum_{j} \sum_{t} \sum_{m} \sum_{k} \lambda_{1} \cdot B_{m} \cdot T_{i j}^{t} \cdot N_{i j, m}^{t} \cdot \psi_{m, k} \cdot x_{i j, k}^{t}, \\
T_{i j}^{t} & =t_{i j} \cdot\left(1+a\left(\frac{q_{i j}^{t}}{C_{i j}}\right)^{b}\right),
\end{aligned}
$$

where $B_{m}$ is the conversion coefficient of truck type $m$ converted to the standard car. $N_{i j, m}^{t}$ denotes number of truck type $m$ passing through route $(i, j)$ at time horizon $t$. $x_{i j, k}^{t}$ is decision variable; $x_{i j, k}^{t}=1$ means that the truck $k$ passes through route $(i, j)$ at time horizon $t$; otherwise, $x_{i j, k}^{t}=0 . \psi_{m, k}$ is decision variable; $\psi_{m, k}=1$ means that the truck $k$ belongs to type $m$; otherwise, $\psi_{m, k}=0 . \lambda_{1}$ is the cost conversion factor of traffic congestion.

$T_{i j}^{t}$ is road resistance function for route $(i, j)$ at time horizon $t$. We use the BPR (Bureau of Public Road) function in this paper. $t_{i j}$ denotes free flow time of a truck passing route $(i, j) . q_{i j}^{t}$ expresses the traffic flows of route $(i, j)$ at time horizon $t$. With the help of transportation big data information platform, we can obtain real-time traffic flows for urban road. $C_{i j}$ is the capacity of the route $(i, j) . a$ and $b$ are the parameters of road resistance function, respectively.

3.2.2. Exhaust Emissions Cost. Different types of trucks produce unequal exhaust emission volume, and the exhaust emissions of a truck are also different at different speeds. The classification of logistics transportation truck types is based on the MOBILE model. The trucks are divided into four types: Heavy-Duty Gasoline Vehicles (HDGV), Light-Duty Diesel Vehicles (LDDV), Light-Duty Diesel Trucks (LDDT), and Heavy-Duty Diesel Vehicles (HDDV). The total masses of all the logistics transportation trucks are above 3.5 tons.

Emissions such as CO, NOx, hydrocarbon, and PM are emitted when the truck is working. In this paper, we choose the $\mathrm{CO}$ and $\mathrm{NOx}$ for the measure of truck exhaust emissions. Exhaust emission factors are determined using the MOBILE6.2 model, which is an emission factor model introduced by the US Environmental Protection Agency [39].

With the help of the Vehicle Mass Analysis System (VMAS), we have obtained exhaust emission factors at different speeds, which can be seen in Table 1. Then, by using the least-squares method to obtain the velocity-emission curve, we can gain the exhaust emission factors at any speed.

Previous studies rarely considered the impact of land use properties on environmental pollution. In fact, different land use properties have different tolerance levels for truck 
TABLE 1: The factors of exhaust emission at different speeds $(\mathrm{g} / \mathrm{km})$.

\begin{tabular}{lccccc}
\hline Speed & Emissions & HDGV & LDDV & LDDT & HDDV \\
\hline \multirow{2}{*}{10} & CO & 69.01 & 25.88 & 27.45 & 31.30 \\
& NOx & 2.37 & 24.71 & 25.41 & 31.58 \\
15 & CO & 53.74 & 18.09 & 20.15 & 24.89 \\
& NOx & 2.44 & 21.66 & 23.33 & 28.24 \\
20 & CO & 42.67 & 14.74 & 17.61 & 20.14 \\
& NOx & 2.52 & 20.35 & 22.19 & 25.53 \\
25 & CO & 34.42 & 13.11 & 14.74 & 16.49 \\
& NOx & 2.59 & 18.72 & 21.01 & 23.34 \\
30 & CO & 28.71 & 10.20 & 11.67 & 13.91 \\
& NOx & 2.66 & 16.60 & 18.42 & 21.73 \\
35 & CO & 24.19 & 8.08 & 9.65 & 11.80 \\
& NOx & 2.74 & 14.30 & 16.24 & 20.43 \\
40 & CO & 30.16 & 6.11 & 8.40 & 10.20 \\
& NOx & 2.82 & 12.72 & 14.08 & 19.48 \\
\hline
\end{tabular}

exhaust emissions. People can hardly tolerate the pollution caused by the operation of trucks in residential areas and commercial areas. On the contrary, people's response is relatively mild in industrial areas and logistics storage areas. Therefore, we innovatively propose the index of regional pollution tolerance, defined as $D$.

$$
D_{f}^{t}=U_{f} \cdot G_{f}^{t},
$$

where $D_{f}^{t}$ is the tolerance of area $f$ at time horizon $t$. $U_{f}$ denotes the average land price of area $f . G_{f}^{t}$ denotes the vitality of area $f$ at time horizon $t$, which can be expressed by the density of activities people in the area. The total cost of exhaust emissions can be expressed as

$$
\mathrm{EC}=\sum_{i} \sum_{j} \sum_{t} \sum_{m} \sum_{k} \sum_{w} \lambda_{2} \cdot D_{f}^{t} \cdot N_{i j, m}^{t} \cdot L_{i j} \cdot E_{i j, m}^{w}(v) \cdot \psi_{m, k} \cdot x_{i j, k}^{t},
$$

where $L_{i j}$ is the length of the route $(i, j) . E_{i j, m}^{w}(v)$ is the exhaust emission factor of the $w$ th pollutant of type $m$ on route $(i, j)$. It relates to truck's speed. $\lambda_{2}$ is the cost conversion factor of exhaust emissions.

3.2.3. Traffic Safety Cost. Because the weight of the logistics transportation truck is heavy when it is full of cargo, it becomes difficult to brake and slow down when the truck is traveling at a fast speed. In addition, there is a large possibility of rollover owing to the high gravity center. Therefore, trucks would increase the risk of traffic accidents when they are traveling on urban roads. The occurrence of accident rates is highly correlated with regional population density. We define SC as the traffic safety cost:

$$
\mathrm{SC}=\sum_{i} \sum_{j} \sum_{t} \sum_{m} \sum_{k} \sum_{f} \lambda_{3} \cdot \frac{P_{f} \cdot r_{f}^{t}}{A_{f}} \cdot s_{m} \cdot x_{i j, k}^{t} \cdot y_{i j}^{f} \text {, }
$$

where $P_{f}$ and $A_{f}$ denote the total population and acreage of area $f$, respectively. $r_{f}$ is travel rate of area $f$ at time horizon $t$. $s_{m}$ is the safety factor of type $m . y_{i j}^{f}$ is decision variable; $y_{i j}^{f}=$ 1 means that the route $(i, j)$ belongs to the area $f$; and otherwise, $y_{i j}^{f}=0 . \lambda_{3}$ is the cost conversion factor of traffic safety.

3.2.4. Logistics Transportation Cost. The logistics transportation cost mainly considers the fuel cost and time cost. LC is used to express the logistics transportation cost:

$$
\mathrm{LC}=\sum_{i} \sum_{j} \sum_{t} \sum_{m} \sum_{k}\left(\lambda_{4} \cdot T_{i j}^{t}+L_{i j} \cdot \sigma_{m}\right) \cdot x_{i j, k}^{t},
$$

where $\sigma_{m}$ is the fuel cost per kilometer and $\lambda_{4}$ is the cost conversion factor of time.

In summary, we establish the truck path optimization model considering environmental impact (TPOM-EI) which minimizes social costs and logistics transportation costs. The objective function of the model is as follows.

$\min Z=\mathrm{TC}+\mathrm{EC}+\mathrm{SC}+\mathrm{LC}$

$$
=\sum_{i} \sum_{j} \sum_{m} \sum_{t} \sum_{k} \sum_{w} \sum_{f}\left(\frac{\lambda_{1} \cdot B_{m} \cdot T_{i j}^{t} \cdot N_{i j, m}^{t} \cdot \psi_{m, k}+\lambda_{2} \cdot D_{f}^{t} \cdot N_{i j, m}^{t} \cdot L_{i j} \cdot E_{i j, m}^{w}(v) \cdot \psi_{m, k}+\lambda_{3} \cdot P_{f} \cdot r_{f}^{t} \cdot s_{m} \cdot y_{i j}^{f}}{A_{f}+\lambda_{4} \cdot T_{i j}^{t}+L_{i j} \cdot \sigma_{m}}\right) \cdot x_{i j, k}^{t},
$$

subject to

$$
\begin{gathered}
\sum_{i} x_{i j, k}^{t} \leq 1, \\
\sum_{j} x_{i j, k}^{t} \leq 1, \\
\sum_{m} N_{i j, m}^{t}=\sum_{k} x_{i j, k}^{t}, \\
x_{i j, k}^{t}+x_{j i, k}^{t} \leq 1, \quad i<j, \forall i, j .
\end{gathered}
$$

It is shown from equation (7) that each subfactor considers time horizon with the decision variable $x_{i j, k}^{t} . \lambda_{1}, \lambda_{2}$, and $\lambda_{3}$ are used to unify cost conversion of factors.

Equations (8) and (9) restrict each node to be accessed only once. Equation (10) guarantees that truck traffic is conserved. Equation (11) ensures that each route can only be visited once.

\section{Model Solution}

The TPOM-EI proposed in this paper belongs to the NPhard problem. Therefore, the model is solved by a heuristic 
algorithm - ant colony optimization (ACO) algorithm. ACO algorithm is a simulation evolution algorithm based on ant foraging behavior proposed by Dorigo et al. [40]. When the ants forage, although ants cannot communicate with each other, each ant will leave a certain concentration of pheromone on the path it has traveled. The next ants would have a high probability to choose the path with more pheromone. Ants use the indirect communication method to achieve efficient coordination of group behavior. Under collective cooperation, the ant colony will search blindly and randomly from the beginning and gradually find and stabilize the optimal foraging path state [41]. Compared with other heuristic algorithms, ACO algorithm has stronger global search ability and better distributed parallel mechanism and self-learning ability. Moreover, the efficient cooperation between ants through pheromones also improves the ability to find the optimal solution.

4.1. Parameter Definition. The main parameter definitions are given as shown in Table 2.

\subsection{Moving and Pheromone Update Strategy}

4.2.1. Moving Strategy. Moving strategy is an important part of ACO algorithm, which is a basis for ants to find all possible paths. Each ant selects the next node according to pheromone and heuristic information. In the initial state, the pheromone of each route is constant $C$, and the heuristic information is the reciprocal of the length of the route. The heuristic information reflects the expected degree of the ant to move between any two nodes. Then the probability that the $k$ th ant moves from node $i$ to node $j$ can be expressed as

$$
p_{i j}^{k}= \begin{cases}\frac{\tau_{i j}^{\alpha} \cdot \eta_{i j}^{\beta}}{\sum_{h \notin \mathrm{tab} u_{i}^{k}} \tau_{i h}^{\alpha} \cdot \eta_{i h}^{\beta},} & j \notin \operatorname{tab} u_{i}^{k}, \\ 0, & \text { otherwise, }\end{cases}
$$

where $\alpha$ and $\beta$ control the weight of pheromone and heuristic information in the moving strategy. Generally, paths with higher pheromone concentrations and shorter lengths are more likely to be selected. But this does not mean that, after the $k$ th ant arrives at node $i$, it must select node $j$ whose route has the largest $p_{i j}^{k}$ as the next access node, because this would lose the opportunity to find some better solutions, thereby reducing the global search ability of the algorithm.

In order to ensure that each path search can randomly select nodes with a high probability, we combine the roulette method to improve the moving strategy. This kind of moving strategy is called a pseudorandom proportional moving strategy. The ant chooses the next node $j$ to be visited, which can be expressed as

$$
j= \begin{cases}\underset{h \notin \operatorname{tab} u_{i}^{k}}{\arg \max }\left\{\tau_{i h}^{\alpha} \cdot \eta_{i h}^{\beta}\right\} & R \leq R_{0}, \\ S, & R>R_{0},\end{cases}
$$

where $R$ is a random number obeying $[0,1]$ uniform distribution. $R_{0}$ is a preset parameter between 0 and 1 . When $R \leq R_{0}$, the ant will definitely choose the node corresponding to the maximum moving probability. When $R>R_{0}$, the ant will choose the node according to the principle of roulette. $S$ is the set of access nodes. A node is randomly selected according to the probability distribution given by equation (12). The specific steps to determine $S$ are as follows:

Step 1: $\forall j \notin$ tab $u_{i}^{k}$, calculate moving probability $p_{i j}^{k}$ and cumulative probability $q_{i}^{k}(n)$, where $q_{i}^{k}(n)$ is equal to the sum of all previous $n$ moving probabilities.

Step 2: Generate a uniformly distributed random number $r$ in $[0,1]$.

Step 3: Find two cumulative probabilities $q_{i}^{k}(n-1)$ and $q_{i}^{k}(n)$ which fulfill the condition $q_{i}^{k}(n-1) \leq r \leq q_{i}^{k}(n)$. The node sets corresponding to $q_{i}^{k}(n)$ and $q_{i}^{k}(n-1)$ are $N_{1}$ and $N_{2}$, respectively. We would determine $S=N_{1} \cap N_{2}$.

4.2.2. Pheromone Update Strategy. Since pheromone and heuristic information will jointly guide the ant behavior, the heuristic information is always equal to the inverse of the length of the route, and the pheromone will change when an ant passes through. If there is too much pheromone remained on the route, the effect of the heuristic information on the ants' choice of path would be greatly reduced.

Therefore, in order to ensure that the effectiveness of the heuristic information will not be affected by excessive pheromone, the pheromone on all routes needs to be updated in ant colony activities. The update methods contain local update and global update. The local update means that the ant should update the pheromone every time it passes through a route. The global update means that the ant updates the pheromone of all the routes that it has passed through. The experiments show that the global update strategy is more effective and can better reflect the overall advantages and disadvantages of the solution. Therefore, we choose the global pheromone update strategy in this paper.

$\Delta \tau_{i j}^{k}$ represents the total amount of pheromone released by the $k$ th ant on route $(i, j)$. Then, after $K$ ants complete the traversal search, the total amount of pheromone changes on each route $(i, j)$ which can be seen in the following equation:

$$
\begin{aligned}
\Delta \tau(i, j) & =\sum_{k} \Delta \tau_{i j}^{k}, \\
\Delta \tau_{i j}^{k} & = \begin{cases}\frac{\rho \cdot Q}{\mathrm{TC}_{k}}, & \text { ant } k \text { pass route }(i, j), \\
0, & \text { otherwise, }\end{cases}
\end{aligned}
$$

where $Q$ is a constant, indicating the total amount of pheromone released by all ants on the path after the traversal search. $\mathrm{TC}_{k}$ represents the total cost generated by the $k$ th ant. $\rho$ denotes the pheromone retention capacity. The pheromone of the updated route $(i, j)$ can be expressed as 
TABLE 2: The sets, indices, and parameters used for solving the TPOM-EI.

\begin{tabular}{lc}
\hline Parameter & Definition \\
\hline$K$ & Set of the ant colony \\
Iter & Index of ants \\
NC & Maximum number of iterations \\
$\tau_{i j}$ & Iteration counter, NC $\leq$ Iter \\
$\eta_{i j}$ & Pheromone concentration on route $(i, j)$ \\
$\alpha$ & Heuristic information concentration on route $(i, j)$, \\
$\beta$ & Weight of pheromone concentration \\
$\rho$ & Weight of heuristic pheromone concentration \\
$Q$ & Pheromone retention capacity \\
$S$ & Total amount of pheromone \\
TC & Set of access nodes \\
tab $u_{i}^{k}$ & Total cost of the $k$ th ant \\
tab $u_{i+}^{k}$ & Set of nodes allowed to be visited by the $k$ th ant at \\
$p_{i j}^{k}$ & node $i$ \\
$q_{i}^{k}(n)$ & Probability of the $k$ th ant moving from node $i$ to node \\
$R$ & Sum of all previous $n$ moving probabilities \\
$R_{0}$ & Random number obeying $[0,1]$ uniform distribution \\
$\Delta \tau_{i j}^{k}$ & Total amount of pheromone released by the $k$ th ant on \\
$\Delta \tau(i, j)$ & route $(i, j)$ \\
$Z_{k}$ & Total gap of pheromone on route $(i, j)$ \\
\hline & Total cost of travel for ant
\end{tabular}

$$
\tau_{i j}= \begin{cases}\rho \cdot \tau_{i j}+\Delta \tau(i, j), & \exists \text { ant } k \text { pass route }(i, j) \\ \rho \cdot \tau_{i j}, & \text { otherwise. }\end{cases}
$$

4.3. The Optimization Process of ACO Algorithm. This section presents a multicriteria hierarchical ant colony optimization algorithm to solve the TPOM-EI in the proposed logistics transportation network. The steps of ACO algorithms are given in Table 3.

\section{Case Study}

Xiqing Economic and Technological Development Zone of Tianjin city (XQ-EDZ) was mainly dominated by the logistics industry in the early years with numerous logistics stations. With the acceleration of urbanization, the population of XQ-EDZ has been increasing and regional functions have been continuously improved. However, the traffic conditions are very poor due to the high proportion of logistics transportation trucks. A few years ago, the local government department adopted measures to prohibit truck traffic on several major roads, but the policy did not achieve the expected results and even worsened traffic condition and safety. Therefore, we use XQ-EDZ as a case study to demonstrate the applicability of the proposed algorithm. Several experiments are performed to validate the effectiveness of the proposed model.

Figure 2 shows the core area of Xiqing Economic and Technological Development Zone with $242.63 \mathrm{~km}^{2}$ and 134 roads which contain 18 highways, 75 main roads, and 41
TABLE 3: The steps of ACO algorithms.

ACO algorithm
Inputs: O-D nodes (o, d) and parameters of $K, N C, \alpha, \beta, \rho, Q$
The arrival rate for each truck and the travel time distribution for
each area
The real-time traffic flow on each route
Returns: optimal truck paths
Step 1. Initialization:
Initialize border nodes and tabu list
For each $k \in K$ do
Let border node $o$ be the origin location
$i \longleftarrow o / i \in$ tub $u_{o+}^{k}$
End for
Step 2. Path selection:
For $i \longleftarrow 1$ to $|K|-1$ do
For each $k \in K$ do
Choose the next node $j$ according to the equation mentioned
Add edge $i, j$ to tab $u^{k}$
$j \longleftarrow i$
End for
End for
Step 3 . Path extension:
Compute $Z_{k} \forall k \in K / Z_{k}$ is the total cost of travel for ant $k$
Update $\Delta \tau(i, j)$ and $\tau_{i j}$ according to the equation mentioned
While not end condition do
Go to Step 2
End while
Print optimal truck paths

secondary roads. Different land use properties are represented by different colors. Blue lines are traffic network and the internal roads of each area are not shown in the figure. There are 10 logistics stations in the zone, which are represented by A to J. Logistics transportation trucks enter or exit from the direction of (1) to (9). The white arrows indicate the entrance of each logistics station. To simplify the problem, we set the arrival rate of trucks at each logistics station based on the size and source, which is shown in Table 4.

Through statistical data of public transportation station provided by Tianjin Rail Transit, we have obtained the people's travel time distribution in different types of areas on workday. This can be seen in Figure 3. The figure shows that different types of areas have significant differences in travel time distribution. Commercial area has the maximum number of trips compared with other areas and it has three peak hours in a day, while the residential area only has two peak hours.

We solve the TPOM-EI model by ACO algorithms. Properly choosing the value range of $\alpha$ and $\beta$ can get better search results; we adopted the recommended values in the literature [42]. The parameter values are as follows. $K=200$, Iter $=500, \alpha=3, \beta=1.5$, and $\rho=0.75, \lambda_{1}, \lambda_{2}, \lambda_{3}, \lambda_{4}$ are the cost conversion factors, which could be defined by the ratio of per capita GDP to social welfare value in Tianjin city.

In this paper, we divide one day into 24 time periods. So, we would gain $24 * 9 * 10=2160$ kinds of path optimization results. In order to highlight the TPOM-EI model, we screen out valuable results in massive results. Taking the model validity into consideration, we pick out 12 typical scenarios 


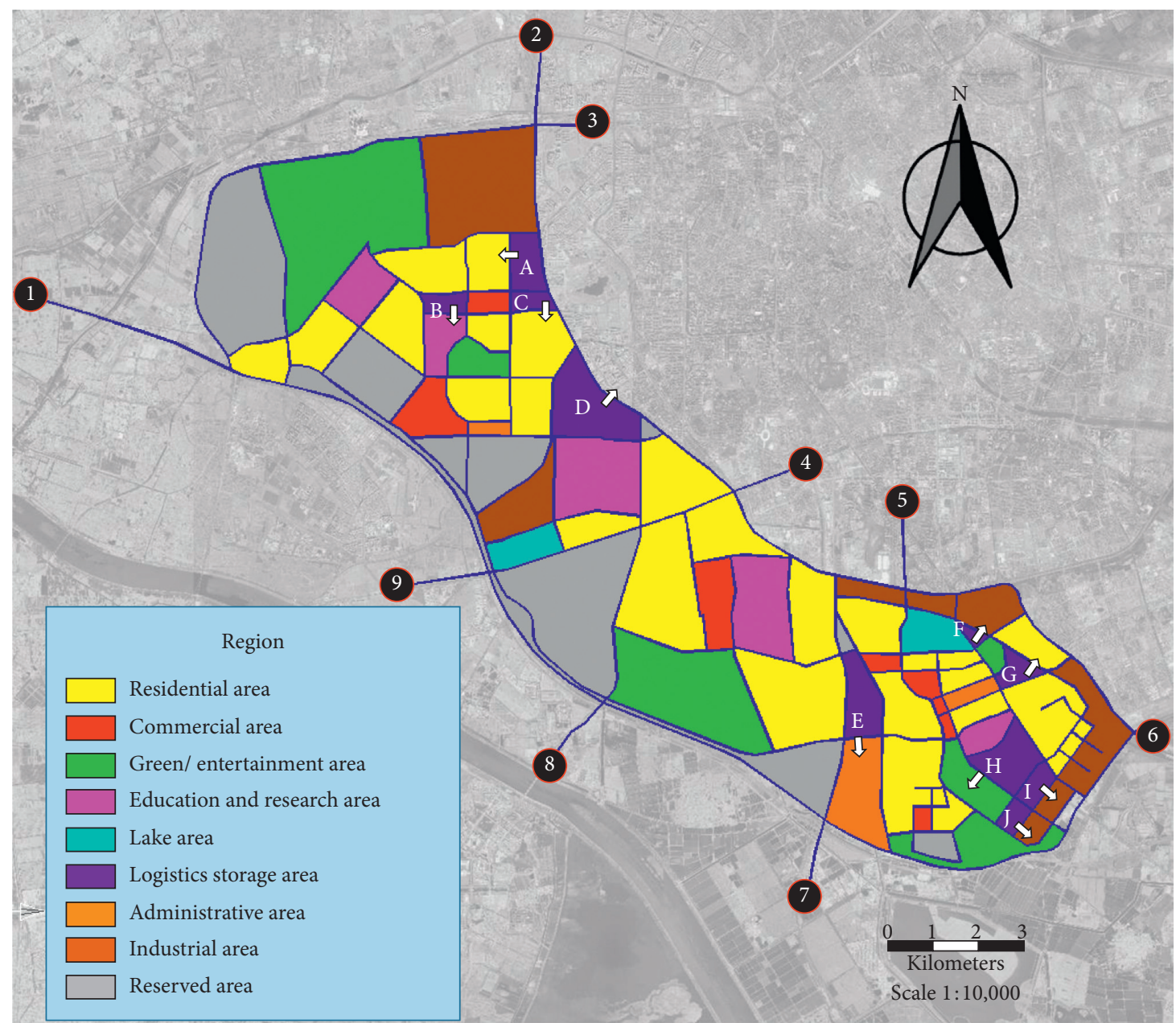

Figure 2: Core area of Xiqing Economic and Technological Development Zone.

TABLE 4: The arrival rate of trucks at each logistics station.

\begin{tabular}{lccccccccc}
\hline Arrival rate (veh/h) & (1) & (2) & (3) & (4) & (5) & (6) & (7) & (8) & (9) \\
\hline $\mathrm{A}$ & 15 & 15 & 20 & 12 & 3 & 15 & 5 & 5 & 6 \\
$\mathrm{~B}$ & 12 & 10 & 15 & 20 & 5 & 12 & 5 & 10 & 5 \\
$\mathrm{C}$ & 10 & 10 & 12 & 8 & 5 & 10 & 2 & 6 & 12 \\
$\mathrm{D}$ & 15 & 12 & 10 & 10 & 8 & 10 & 12 & 15 & 15 \\
$\mathrm{E}$ & 15 & 18 & 5 & 8 & 15 & 25 & 15 & 2 & 10 \\
$\mathrm{~F}$ & 8 & 10 & 2 & 3 & 15 & 5 & 6 & 4 & 2 \\
$\mathrm{G}$ & 10 & 12 & 5 & 2 & 10 & 8 & 5 & 6 & 5 \\
$\mathrm{H}$ & 20 & 15 & 10 & 5 & 6 & 12 & 15 & 8 & 6 \\
$\mathrm{I}$ & 10 & 12 & 2 & 8 & 8 & 6 & 12 & 3 & 3 \\
$\mathrm{~J}$ & 10 & 10 & 2 & 2 & 6 & 5 & 12 & 2 & 6
\end{tabular}

that have different results solved by the shortest path model and the proposed model in this paper. Table 5 shows the truck path optimization results in off-peak hour (2 pm-3 $\mathrm{pm})$.

It can be seen from Table 5 that the TPOM-EI model has increased the travel lengths by $6.03 \%$ to $25.11 \%$ compared with the shortest path model. Although the travel length is increased, the total social cost is reduced. By further analysis, we consider adding time variables to optimize the truck's travel path in real time and join the arrival rates at different time horizons. The lengths of truck path optimization at different time horizons are shown in Table 6.

We can see from Table 6 that the optimization of logistics transportation truck paths has significant change for some scenarios at different time horizons in workdays, such as (2)-J, (3)-I, and (8)-A. Because the social costs of these logistics transportation truck paths are sensitive, in other words, these paths have a large impact on social costs, the logistics transportation truck paths are constantly adjusted as the activity of each area changes. However, we also find some scenarios that are not sensitive to the change of time horizons (e.g., (3)-B, (7)-D, and (7)-F). The increased cost of trucking logistics transportation is far higher than the social cost reduction caused by traffic congestion, environmental impact, and traffic safety.

We analyze the optimized results of logistics transportation truck path further by contrasting the peak hour and off-peak hour. We find that the length of an optimized path during peak hours is farther than that during off-peak hours. Because the social cost of some roads increases during peak hours, trucks are forced to find other paths which increases logistics transportation costs. Besides, we also find an interesting result that the length of optimized path during peak hours is less than that during off-peak hours (e.g., (4)-H and (6)-E). It seems to be contrary to our expectations. 


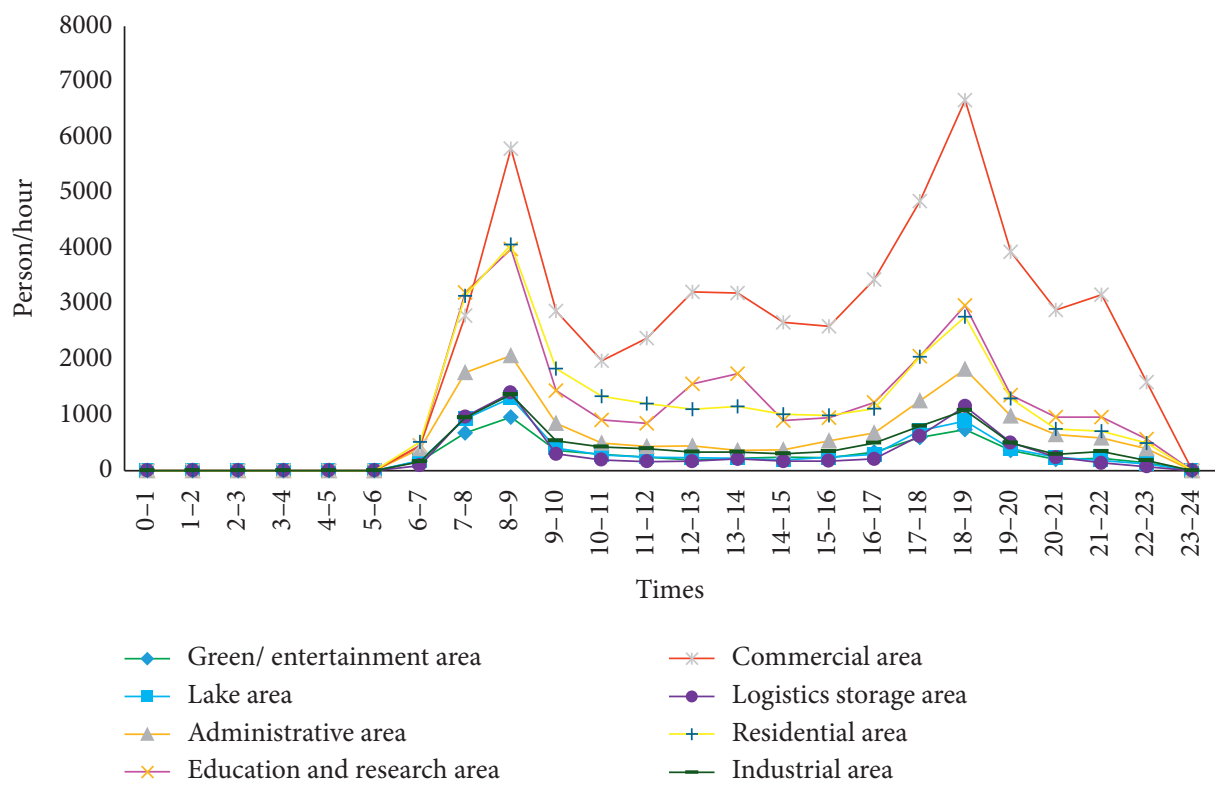

Figure 3: People's travel time distribution in different types of areas on workday.

TABle 5: Truck path optimization results in off-peak hour.

\begin{tabular}{|c|c|c|c|c|c|}
\hline Scenarios & Path direction & Logistics stations & Shortest lengths $(\mathrm{km})$ & Optimized lengths $(\mathrm{km})$ & Gap (\%) \\
\hline 1 & (1) & $\mathrm{H}$ & 28.17 & 30.84 & 9.48 \\
\hline 2 & (2) & $\mathrm{J}$ & 26.41 & 29.88 & 13.14 \\
\hline 3 & (3) & $\mathrm{B}$ & 6.70 & 7.74 & 15.52 \\
\hline 4 & (3) & I & 29.52 & 31.30 & 6.03 \\
\hline 5 & (4) & $\mathrm{H}$ & 13.69 & 15.44 & 12.78 \\
\hline 6 & (5) & G & 4.85 & 5.96 & 22.89 \\
\hline 7 & (6) & B & 22.47 & 24.02 & 6.90 \\
\hline 8 & (6) & $\mathrm{E}$ & 13.26 & 16.59 & 25.11 \\
\hline 9 & (7) & $\mathrm{D}$ & 20.04 & 23.68 & 18.16 \\
\hline 10 & (7) & $\mathrm{F}$ & 15.08 & 17.91 & 18.77 \\
\hline 11 & (8) & A & 17.70 & 19.56 & 10.51 \\
\hline 12 & (9) & $\mathrm{C}$ & 14.05 & 15.38 & 9.47 \\
\hline
\end{tabular}

TABLE 6: The lengths of truck path optimization at different time horizons.

\begin{tabular}{|c|c|c|c|c|c|c|c|c|c|c|c|c|}
\hline Time horizons & (1)- $\mathrm{H}$ & (2) $-J$ & (3) $-\mathrm{B}$ & (3)-I & (4) $-\mathrm{H}$ & (5) $-\mathrm{G}$ & (6) $-B$ & (6)-E & (7)-D & (7)-F & (8)-A & (9)-C \\
\hline $0-1$ & 28.17 & 26.41 & 6.70 & 29.52 & 13.69 & 4.85 & 22.47 & 13.26 & 20.04 & 15.08 & 17.70 & 14.05 \\
\hline $1-2$ & 28.17 & 26.41 & 6.70 & 29.52 & 13.69 & 4.85 & 22.47 & 13.26 & 20.04 & 15.08 & 17.70 & 14.05 \\
\hline $2-3$ & 28.17 & 26.41 & 6.70 & 29.52 & 13.69 & 4.85 & 22.47 & 13.26 & 20.04 & 15.08 & 17.70 & 14.05 \\
\hline $3-4$ & 28.17 & 26.41 & 6.70 & 29.52 & 13.69 & 4.85 & 22.47 & 13.26 & 20.04 & 15.08 & 17.70 & 14.05 \\
\hline 4-5 & 28.17 & 26.41 & 6.70 & 29.52 & 13.69 & 4.85 & 22.47 & 13.26 & 20.04 & 15.08 & 17.70 & 14.05 \\
\hline $5-6$ & 28.17 & 26.41 & 6.70 & 29.52 & 13.69 & 4.85 & 22.47 & 13.26 & 20.04 & 15.08 & 17.70 & 14.05 \\
\hline $6-7$ & 29.28 & 27.60 & 6.70 & 30.47 & 15.44 & 5.96 & 24.02 & 16.59 & 20.04 & 17.91 & 19.56 & 15.38 \\
\hline $7-8$ & 30.25 & 30.52 & 7.74 & 31.30 & 15.44 & 6.22 & 24.02 & 15.25 & 23.68 & 17.91 & 21.02 & 17.63 \\
\hline $8-9$ & 30.84 & 31.75 & 7.74 & 32.96 & 13.69 & 6.73 & 25.91 & 15.25 & 23.68 & 17.91 & 21.02 & 17.63 \\
\hline $9-10$ & 30.84 & 31.75 & 7.74 & 31.30 & 13.69 & 6.73 & 25.91 & 15.25 & 20.04 & 17.91 & 19.56 & 15.38 \\
\hline $10-11$ & 30.84 & 30.52 & 7.74 & 30.47 & 15.44 & 6.22 & 24.02 & 16.59 & 20.04 & 17.91 & 19.56 & 15.38 \\
\hline $11-12$ & 30.84 & 29.88 & 7.74 & 31.30 & 15.44 & 5.96 & 24.02 & 16.59 & 20.04 & 17.91 & 19.56 & 15.38 \\
\hline $12-13$ & 30.84 & 29.88 & 7.74 & 30.47 & 15.44 & 5.96 & 24.02 & 16.59 & 20.04 & 17.91 & 19.56 & 15.38 \\
\hline $13-14$ & 32.76 & 30.52 & 7.74 & 31.30 & 15.44 & 6.22 & 24.80 & 15.25 & 20.04 & 17.91 & 19.56 & 15.38 \\
\hline $14-15$ & 30.25 & 27.60 & 7.74 & 30.47 & 15.44 & 5.96 & 24.80 & 16.59 & 20.04 & 17.91 & 19.56 & 15.38 \\
\hline $15-16$ & 30.25 & 27.60 & 7.74 & 32.96 & 15.44 & 5.96 & 24.02 & 16.59 & 20.04 & 17.91 & 19.56 & 15.38 \\
\hline $16-17$ & 32.76 & 30.52 & 7.74 & 32.96 & 13.69 & 5.96 & 25.91 & 16.59 & 23.68 & 17.91 & 19.56 & 17.63 \\
\hline $17-18$ & 32.76 & 31.75 & 7.74 & 32.96 & 13.69 & 6.73 & 25.91 & 15.25 & 23.68 & 17.91 & 21.02 & 17.63 \\
\hline $18-19$ & 32.76 & 31.75 & 7.74 & 32.96 & 13.69 & 6.73 & 25.91 & 15.25 & 23.68 & 17.91 & 21.02 & 17.63 \\
\hline $19-20$ & 32.76 & 31.75 & 7.74 & 32.96 & 13.69 & 6.73 & 25.91 & 15.25 & 23.68 & 17.91 & 21.02 & 17.63 \\
\hline $20-21$ & 30.25 & 31.75 & 7.74 & 31.30 & 13.69 & 6.73 & 25.91 & 15.25 & 23.68 & 17.91 & 21.02 & 15.38 \\
\hline $21-22$ & 32.76 & 31.75 & 7.74 & 31.30 & 15.44 & 6.22 & 24.80 & 16.59 & 23.68 & 17.91 & 19.56 & 15.38 \\
\hline $22-23$ & 29.28 & 27.60 & 7.74 & 30.47 & 15.44 & 5.96 & 24.02 & 16.59 & 23.68 & 17.91 & 19.56 & 15.38 \\
\hline $23-24$ & 28.17 & 26.41 & 6.70 & 29.52 & 13.69 & 4.85 & 22.47 & 13.26 & 20.04 & 15.08 & 17.70 & 14.05 \\
\hline
\end{tabular}


TABLE 7: Comparison of optimization results between two strategies.

\begin{tabular}{lcccccc}
\hline Strategy & $\begin{array}{c}\text { Computing } \\
\text { time }(\mathrm{s})\end{array}$ & $\begin{array}{c}\text { Total running } \\
\text { distance }(\mathrm{km})\end{array}$ & $\begin{array}{c}\text { Total exhaust } \\
\text { emission }(\mathrm{kg})\end{array}$ & $\begin{array}{c}\text { Average traffic safety } \\
\text { factor }(-)\end{array}$ & $\begin{array}{c}\text { Average traffic } \\
\text { congestion factor }(-)\end{array}$ & $\begin{array}{c}\text { Objective value } \\
(\text { Yuan })\end{array}$ \\
\hline S1 & 386 & $3,117.5$ & $1,210.825$ & 0.708 & 0.527 & $562,428.30$ \\
S2 & 294 & $2,448.0$ & $1,028.645$ & 0.426 & 0.640 & $583,954.62$ \\
Gap (\%) & -31.29 & -27.35 & -17.71 & 66.20 & 21.44 & 3.83 \\
\hline
\end{tabular}

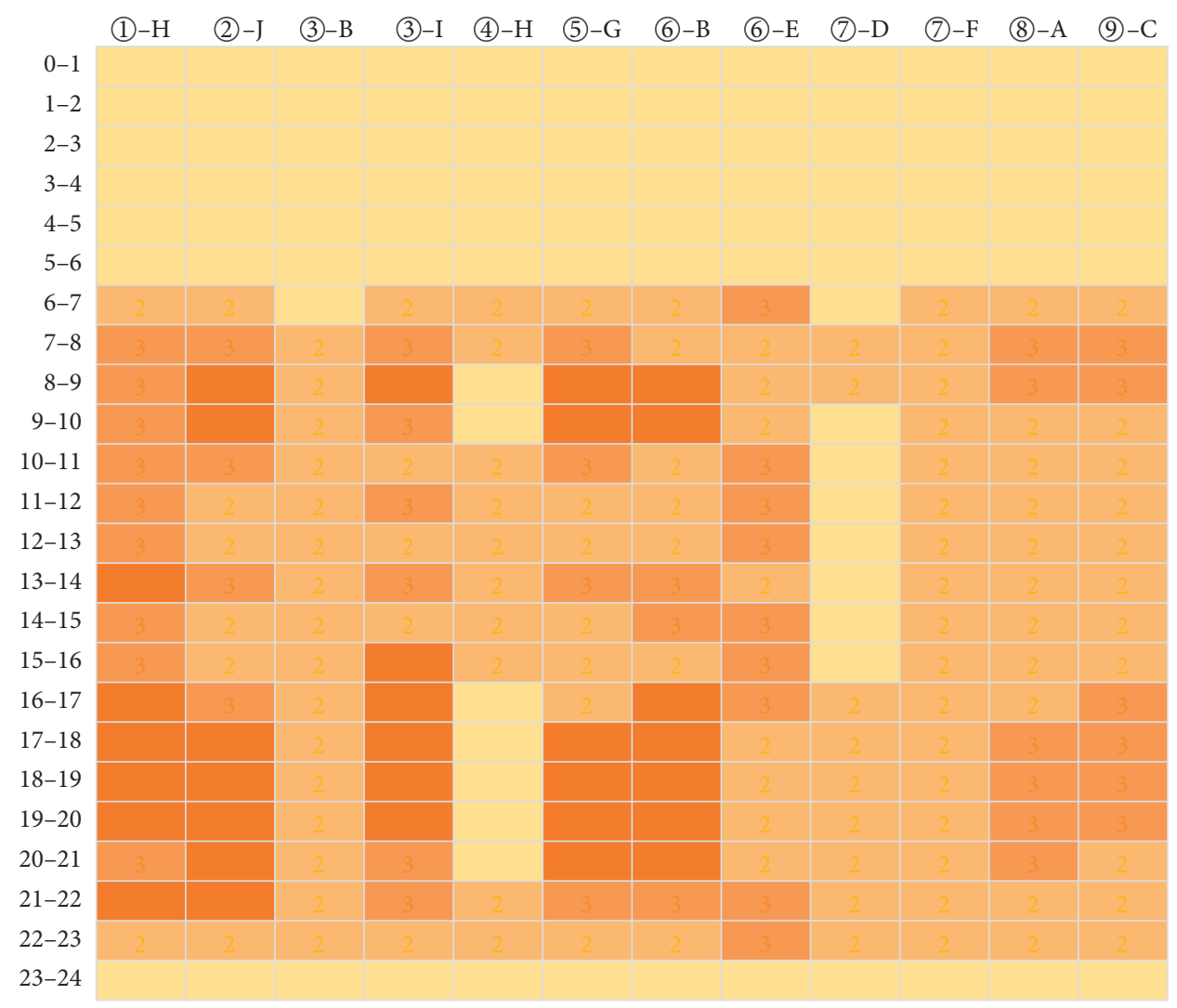

FIgURE 4: The color chart of truck path lengths at different time horizons.

Then we conducted an in-depth analysis of these optimization paths. We have found that the social costs of some roads during peak hours have sharply risen, even higher than the social costs on the shortest path, due to the differences distribution of travel rate in the spatiotemporal network. Therefore, there is a phenomenon of reducing the logistics transportation costs while further reducing the social costs.

By further analysis of the superiority of our model, we have optimized the operations of logistics transportation truck by using a method (S2 strategy) which just considered shortest path to the method (S1 strategy) that considered environmental impact we presented in this paper. The results are shown in Table 7. Compared with S2 strategy, S1 strategy increases the time dimension, which causes the computing time to be longer, and the time complexity of the proposed model is increased by $31.29 \%$. Although the total running distance and exhaust emission of S1 strategy are more than those of S2 strategy, the objective value of S1 strategy is better than that of S2 strategy. The objective value reduces from $562,428.30$ to $583,954.62$ because the social costs are greatly reduced with the gap of average traffic safety factor increasing by $66.20 \%$, while the gap of average traffic congestion factor reduced by $21.44 \%$. The results have shown that S1 strategy has a significant improvement over S2 strategy.

Finally, we summarize the similar features of truck path optimization at different time horizons. We cluster the lengths of path optimization by normalization and display it by color chart seen in Figure 4 . The result shows three significant time horizons, trough hours (23 pm-6 am), offpeak hours (10 am-16 pm), and peak hours (6 am-10 am and $16 \mathrm{pm}-22 \mathrm{pm})$, respectively. During the trough hours, there is no need to restrict logistics transportation truck path and the truck can choose the shortest path to reduce transportation cost. During the off-peak hours, traffic management department takes control measures on important roads and logistics transportation trucks still have several choices to plan their paths. However, traffic management department should pay more attention to social costs to restrict logistics transportation truck traveling in environmentally sensitive areas during the peak hours. Our research not only could provide a quantitative basis for 
related policy development but also could help truck drivers choose optimal paths in real time. Moreover, it might provide theoretical guidance for the location of logistics stations and the planning of land use.

\section{Conclusions}

This paper has presented a truck path optimization model considering environmental impact (TPOM-EI), which is solved by heuristic algorithm, ant colony optimization (ACO) algorithm. Transportation costs and social costs are taken into consideration for optimizing the path of logistics transportation truck. At the same time, the proposed model has added to the time factor and gains real-time truck path at different time horizons.

A case study using data of Xiqing Economic and Technological Development Zone in Tianjin city (XQ-EDZ) was carried out for a demonstration. In this paper, 12 typical scenarios that have different results solved by the shortest path model and the proposed model have been picked out. Travel lengths of optimal truck path data demonstrated that environmental impact is, indeed, strongly correlated within urban impact areas.

The TPOM-EI model could effectively reduce congestion, thereby reducing pollutant emissions of logistics transportation trucks, even if the total distance of trucks increased. The results also showed that traffic congestion was one of the most important factors of exhaust emission. In addition, we considered the regional land use to reduce the frequency of vehicles operating in sensitive areas, especially densely populated areas, such as commercial areas, residential areas, and green/entertainment areas. These measures could improve air quality and travel safety and promote social welfare effectively.

The results have shown that some paths are sensitive, that is, have a large impact on social costs. The real-time activity of each area can also change the travel path of a truck. In general, the length of optimized path during peak hours is farther than that during off-peak hours because the social cost during peak hours further increases, making trucks look for other paths to reduce social costs, while inevitably increasing logistics transportation costs. Yet this paper also found an interesting result that the length of the optimized path during peak hours is less than that during off-peak hours. The social costs of some roads during peak hours have sharply risen, even higher than the social costs on the shortest path, due to the differences distribution of travel rate in the spatiotemporal network.

Finally, similar features of truck path optimization at different time horizons have been summarized in this paper. The result shows three significant time horizons, trough hours (23 pm-6 am), off-peak hours (10 am-16 pm), and peak hours $(6 \mathrm{am}-10$ am and $16 \mathrm{pm}-22 \mathrm{pm})$, respectively. Traffic management department could adopt different control strategies at different time horizons. Our research could not only provide a quantitative basis for related policy development but also could help truck drivers choose optimal paths in real time. Moreover, it might provide theoretical guidance for the location of logistics stations and the planning of land use.

\section{Data Availability}

The data used to support the findings of this study are available from the corresponding author upon request.

\section{Conflicts of Interest}

The authors declare no conflicts of study regarding this article.

\section{Acknowledgments}

This research was supported by the Ministry of Education of Humanities and Social Science Project (17YJC630034).

\section{References}

[1] D. Tamagawa, E. Taniguchi, and T. Yamada, "Evaluating city logistics measures using a multi-agent model," Procedia-Social and Behavioral Sciences, vol. 2, no. 3, pp. 6002-6012, 2010.

[2] N. Arvidsson, "The milk run revisited: a load factor paradox with economic and environmental implications for urban freight transport," Transportation Research Part A: Policy and Practice, vol. 51, pp. 56-62, 2013.

[3] F. Russo and A. Comi, "City characteristics and urban goods movements: a way to environmental transportation system in a sustainable city," Procedia-Social and Behavioral Sciences, vol. 39, pp. 61-73, 2012.

[4] J. S. E. Teo, E. Taniguchi, and A. G. Qureshi, "Evaluation of load factor control and urban freight road pricing joint schemes with multi-agent systems learning models," Procedia-Social and Behavioral Sciences, vol. 125, pp. 62-74, 2014.

[5] T. Dorothy, A. K. Robert, and G. Neil, "Compliance costs, regulation, and environmental performance: controlling truck emissions in the us," Regulation \& Governance, vol. 2, no. 3, pp. 275-292, 2008.

[6] Y. Yong and W. Jing, "Study of logistics network optimization model considering carbon emissions," International Journal of Systems Assurance Engineering and Management, vol. 8, no. 10, pp. 1-7, 2013.

[7] A. G. Luis, D. L. H. Daniel, and M. Andrés, "Optimization of transport measures to reduce ghg and pollutant emissions through a luti modeling approach," International Journal of Sustainable Transportation, vol. 10, no. 7, 2016.

[8] X. Yang, W. Zhang, J. Fan, J. Yu, and H. Zhao, "Transfers of embodied pm2.5 emissions from and to the north China region based on a multiregional input-output model," Environmental Pollution, vol. 235, pp. 381-393, 2018.

[9] S. John, E. Richard, L. Chris, and H. David, "Reducing australian motor vehicle greenhouse gas emissions," Transportation Research Part A Policy \& Practice, vol. 109, pp. 76-88, 2018.

[10] I. Fernandez-Barcelo and J. M. Campos-Cacheda, "Estimate of social and environmental costs for the urban distribution of goods," Procedia-Social and Behavioral Sciences, vol. 39, pp. 818-830, 2012.

[11] Y. C. Hu, J. S. Shen, and A. L. Huang, "Study on multi-objective optimization benefits of urban distribution in the scenario of urban freight traffic control," Transportation 
System Engineering and Information, vol. 12, no. 6, pp. 119125, 2012.

[12] Y. L. Li, G. Hua, and D. Q. Sun, "Analysis on impact of china's urban freight vehicle traffic management policy on logistics," Transportation Construction and Management, vol. 11, pp. 74-77, 2013.

[13] L. R. Zhang, Y. Yang, H. F. Tan et al., "Analysis and suggestion of impact of restricting urban freight vehicles on urban distribution," China Logistics \& Purchasing, vol. 24, pp. 110-111, 2015.

[14] Y. Yang and X. J. Yang, "Study on travel restriction of urban freight vehicles based on system dynamics," Journal of Highway and Transportation Research and Development, vol. 34, no. 5, pp. 150-158, 2017.

[15] E. Maggi and E. Vallino, "Understanding urban mobility and the impact of public policies: the role of the agent-based models," Research in Transportation Economics, vol. 55, pp. 50-59, 2016.

[16] G. Valerio and M. Edoardo, "Behavioural implications of nonlinear effects on urban freight transport policies: the case of retailers and transport providers in rome," Case Studies on Transport Policy, vol. 4, no. 1, pp. 22-28, 2016.

[17] L. D. Grange and R. Troncoso, "Impacts of vehicle restrictions on urban transport flows: the case of Santiago, Chile," Transport Policy, vol. 18, no. 6, 2011.

[18] F. Heike and J. Wolff, "Impacts of planning and policy strategies on freight flows in urban areas," Transportation Research Procedia, vol. 12, pp. 584-597, 2016.

[19] S. Swamy and D. Baindur, "Managing urban freight transport in an expanding city-case study of Ahmedabad," Research in Transportation Business \& Management, vol. 11, pp. 5-14, 2014.

[20] C. Lammgard and M. Browne, "Night-time deliveries: supply chain dream or policy nightmare?" in Proceedings of the Logistics Research Network Conference, pp. 43-55, Cranfield, England, September 2012.

[21] X. Deng, J. Xu, and B. Wang, "Traffic countermeasures research for Guangzhou city in traffic mode transferring period after "motorcycle forbidden ban" effect," Journal of Transportation Systems Engineering and Information Technology, vol. 9, no. 4, pp. 145-150, 2009.

[22] X. J. Shen, J. Y. Sun, X. Y. Zhang et al., "The influence of emission control on particle number size distribution and new particle formation during China's V-day parade in 2015," Science of the Total Environment, vol. 573, pp. 409-419, 2015.

[23] R. Hesham, A. Kyoungho, and T. Antonio, "Comparison of mobile5a, mobile6, vt-micro, and cmem models for estimating hot-stabilized light-duty gasoline vehicle emissions," Canadian Journal of Civil Engineering, vol. 30, no. 6, pp. 1010-1021, 2003.

[24] E. M. Fujita, D. E. Campbell, B. Zielinska et al., "Comparison of the moves2010a, mobile6.2, and emfac2007 mobile source emission models with on-road traffic tunnel and remote sensing measurements," Journal of the Air \& Waste Management Association, vol. 62, no. 10, pp. 1134-1149, 2012.

[25] K. Ioannis, K. Chariton, G. Dimitrios, D. Panagiota, and N. Leonidas, "Uncertainty and sensitivity analysis of national road transport inventories compiled with copert 4," ProcediaSocial and Behavioral Sciences, vol. 2, no. 6, pp. 7690-7691, 2010.

[26] S. Sugawara and D. A. Niemeier, "Part 2-air quality-how much can vehicle emissions be-reduced? exploratory analysis of an upper boundary using an emissions-optimized trip assignment," Transportation Research Record, vol. 1815, no. 9, 2002.
[27] E. Ericsson, H. Larsson, and K. Brundell-Freij, "Optimizing route choice for lowest fuel consumption - potential effects of a new driver support tool," Transportation Research Part C: Emerging Technologies, vol. 14, no. 6, pp. 369-383, 2006.

[28] A. Kyoungh and R. Hesham, "The effects of route choice decisions on vehicle energy consumption and emissions," Transportation Research Part D. vol. 13, no. 3, pp. 151-167, 2008.

[29] K. Jaeyoung, P. Byungkyu, and L. Jaesup, "Evaluating the impacts of urban corridor traffic signal optimization on vehicle emissions and fuel consumption," Transportation Planning and Technology, vol. 35, no. 2, pp. 145-160, 2011.

[30] E. W. Dijkstra, "A note on two problems in connexion with graphs," Numerische Mathematik, vol. 1, no. 1, pp. 269-271, 1959.

[31] R. W. Floyd, “Algorithm 97: shortest path," Communications of the ACM, vol. 5, 1962.

[32] G. B. Dantzig and J. H. Ramser, "The truck dispatching problem," Management Science, vol. 6, no. 1, pp. 80-91, 1959.

[33] T. Dinh, R. Fukasawa, and J. Luedtke, "Exact algorithms for the chance-constrained vehicle routing problem," Mathematical Programming, vol. 2, pp. 1-34, 2017.

[34] J. Ren, Y. Tian, and T. Sawaragi, “An exact algorithm for the three-dimensional loading capacitated vehicle routing problem," Chain Modelling, vol. 4, pp. 3-4, 2017.

[35] B. Yao, B. Yu, P. Hu, J. Gao, and M. Zhang, “An improved particle swarm optimization for carton heterogeneous vehicle routing problem with a collection depot," Annals of Operations Research, vol. 242, no. 2, pp. 303-320, 2016.

[36] B. Yu, Z.-Z. Yang, and B. Yao, "An improved ant colony optimization for vehicle routing problem," European Journal of Operational Research, vol. 196, no. 1, pp. 171-176, 2009.

[37] B. Yao, P. Hu, X. Lu, J. Gao, and M. Zhang, "Transit network design based on travel time reliability," Transportation Research Part C: Emerging Technologies, vol. 43, pp. 233-248, 2014.

[38] J. Wu, Z. Gao, and H. Sun, "Simulation of traffic congestion with sir model," Modern Physics Letters B, vol. 18, no. 30, pp. 1537-1542, 2004.

[39] Z. Kai and B. Stuart, "Near-road air pollutant concentrations of co and pm2.5: a comparison of mobile6.2/caline4 and generalized additive models," Atmospheric Environment, vol. 44, no. 14, pp. 1740-1748, 2010.

[40] D. Marco, B. Mauro, and S. Thomas, "Ant colony optimization," IEEE Computational Intelligence Magazine, vol. 1, no. 4, pp. 28-39, 2006.

[41] B. Christian and S. Michael, "An ant colony optimization algorithm for shop scheduling problems," Journal of Mathematical Modelling \& Algorithms, vol. 3, no. 3, pp. 285-308, 2004.

[42] S. C. Zhan, J. Xu, and J. Wu, "The optimal selection of related algorithm parameters in ant colony algorithm," Science \& Technology Bulletin, vol. 05, pp. 381-386, 2003. 